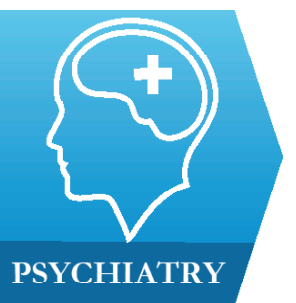

1) Psychiatry Clinic III, Emergency County Hospital Cluj, Cluj-Napoca, Romania

2) Discipline of Medical Psychology, Department of Medical Education, Iuliu Hatieganu University of Medicine and Pharmacy, Cluj-Napoca, Romania
DOI: $10.15386 / \mathrm{mpr}-1300$

Manuscript received: 05.02.2019 Received in revised form: 02.04.2019 Accepted: 13.04.2019

Address for correspondence: valentin.vasadi@gmail.com

\title{
The link between personality dimensions and pain perception in patients with diabetes mellitus or cancer
}

Cătălina-Maria Petrașcu ${ }^{1}$, Valentin-Marcel Vașadi ${ }^{1}$, Răzvan Moisă${ }^{1}$, Marinela Minodora Manea ${ }^{2}$

\begin{abstract}
Background and aims. Diabetes mellitus and cancer are two diseases with a strong psychological impact. Personality traits modulate the perception of pain and the response to disease. The specificity of this study consists of the assessment of personality, pain and emotional impact in the two diseases.
\end{abstract}

Method. The study included 130 participants assigned to 3 groups: patients with diabetes mellitus, patients with cancer disease, and a control group. The personality traits of all the participants were analyzed using Cloninger's Temperament and Character Inventory (TCI). For the evaluation of pain, as well as associated anxiety and depression in the diabetes and cancer groups, the Brief Pain Inventory (BPI) and the Hospital Anxiety and Depression Scale (HADS) were used.

Results. Patients with diabetes mellitus and cancer disease had significantly higher total values on the Harm Avoidance scale $(\mathrm{HA})(\mathrm{p}<0.001)$ and lower total values on the Self-Directedness scale (SD), Reward Dependence scale (RD), Cooperativeness scale $(C O)(p<0.001)$ compared to the control group. Major anxiety was predominant in patients with diabetes mellitus (34\%), and major depression was prevalent in cancer patients $(17.5 \%)$. Patients with diabetes mellitus obtained a significantly higher severity score at BPI scale than patients with cancer disease $(\mathrm{p}=0.03)$.

Conclusions. The existence of a mutual personality profile featuring high levels of Harm Avoidance and low levels of Self-Directedness in patients with diabetes mellitus and cancer who experience pain has great implications for the therapeutic approach. A potential future avenue of management in these diseases may lie in targeting particular personality aspects of chronic pain patients.

Keywords: personality, TCI, pain, diabetes, cancer

\section{Background and aims}

Diabetes mellitus and cancer are two diseases with a strong psychological impact, either through their chronic, disabling nature and negative effect on the quality of life (diabetes) or through their discouraging, frequently fatal evolution (cancer). A common and frequent symptom is pain, especially chronic pain. It is difficult to imagine pain without emotion. Suffering associated with a nociceptive stimulus is reflected in the emotional response, which is useful in adaptive coping mechanisms and more effective in the case of acute pain [1]. However, emotions also contribute to disturbances in the affective area, as a result of chronic pain, in particular. Studies have demonstrated that pain is associated with emotions such as: anger [2], anxiety [3,4], fear $[5,6]$, worry [7]. Psychological factors are strong predictors of pain experience [8] and psychological models are focused on characteristic cognitive, emotional and behavioral patterns that influence the perception of pain. The background in which these patterns develop is represented by personality. Personality traits and other cognitive and emotional patterns play a role in the processing and interpretation of pain experience, and they can be modeled as protective and risk factors for pain [9].

Cloninger's model is composed of 7 dimensions of personality, having the general advantages of dimensionality and retaining at the same time the solid theoretical basis and the empirical support of previous psychobiological models (Eysenck, McCrae), being suitable for the determination of personality traits, as well as for the identification of their pathological 
deviations (personality disorders). The Temperament and Character Inventory (TCI) can be more useful in clinical practice than other personality assessment questionnaires (Minnesota Multiphasic Personality Inventory, NEOPersonality Inventory) because it follows a psychobiological personality model that makes the distinction between the temperament and character dimensions [10]. The different temperament dimensions are defined in terms of basic stimulus-response characteristics. Novelty Seeking (NS) is thought to be related to the behavioral activation system, Harm Avoidance (HA) to the behavioral inhibition system, Reward Dependence (RD) to the behavioral maintenance system, and Persistence $(\mathrm{P})$ to perseverance in behavior despite frustration and fatigue. Of the character dimensions, Self-Directedness (SD) refers to an individual's ability to control and adapt their behavior in accord with chosen goals and values, Cooperativeness (CO) to their tendency towards social tolerance, empathy and compassion, and Self-Transcendence (ST) to their identification with nature and the ability to accept uncertainty [11]. According to Cloninger's theory, temperament represents the "emotional core" of personality, genetically transmitted and considered stable throughout life regardless of the cultural or social context, while character represents the "conceptual core", which develops in stages through the interaction between temperament, socio-cultural environment and life experiences. This theory is supposed to be relevant for the perception of pain because serotonin, the neurotransmitter correlated with Harm Avoidance (HA) [12], is one of the major transmitters in the inhibitory modulation of pain by descending pathways.

Harm avoidance is one of the four independent temperament dimensions that form the "emotional core" of personality. This dimension reflects a neurobiological predisposition to the early emotion of fear and its related automatic behavior, which can be described as inhibition [13]. Individuals with high scores of harm avoidance can easily develop avoidant responses to aversive stimuli, which makes them susceptible to fear [14]. Waddel et al. concluded in their studies that the fear of pain and the behavior associated with this fear is more disabling than the pain itself [15]. The fear-avoidance model states that avoidant movements and activities based on fear are the central mechanism in the development of chronic pain [16]. Depression and disability are consequences of these avoidant behaviors which maintain the pain experience and perpetuate the vicious circle of increasing fear and avoidance [17]. The actual pain behavior may result from an interaction between the perception of somatosensory information, personality characteristics and environmental feedback.

Cloninger's biopsychosocial model was developed and used mainly in the field of psychiatry; few studies use this model in non-psychiatric patients, such as patients with pain $[13,18-21]$.

The main focus of the study is to explore the link between personality dimensions and pain perception in diabetes mellitus and cancer. Specifically,

1. The hypothesis of a higher score in the Harm Avoidance (HA) temperament dimension and a lower score on the Self-Directedness (SD) character scale compared to the control group was advanced.

2. Pain and the associated degree of anxiety and depression were assessed in patients with diabetes mellitus and cancer disease.

\section{Subjects and methods Subjects}

The study was conducted on 130 patients assigned to 3 groups: diabetes (50), cancer (40), and control (40). The first group included 50 diabetic patients admitted to the Clinical Center for Diabetes, Nutrition and Metabolic Diseases ClujNapoca in the period 19.02.2016-11.03.2016. Of these, 13 patients had type 1 diabetes mellitus and 37 patients had type 2 diabetes mellitus. The second group comprised 40 patients admitted to the service of Oncology of the Alba County Emergency Hospital in the period 3.05.2016-6.05.2016. Of these, 29 patients were admitted on a day care basis for chemotherapy, and 11 patients were admitted on an inpatient basis. The third group, representing the control group, included 40 patients with hypertension from Cluj-Napoca selected in the internal medicine department at the free Polyclinic "Holy family", Cluj-Napoca and was only used to compare the groups regarding their personality traits.

Study inclusion criteria: patients diagnosed with type 1 or type 2 diabetes mellitus, with cancer disease regardless of the stage, type of treatment or date of diagnosis; absence of mental retardation, presence of the patient's consent.

Study exclusion criteria: presence of mental retardation, presence of disabling or metastatic pain, presence of high-intensity pain at the moment of evaluation, presence of a diagnosis of anxiety or affective disorder prior to our study, absence of the patient's consent, inadequate mental or physical state, incomplete data because of the patient's discharge or transfer.

The patients were free to withdraw from the study at any time, without prejudice.

The study protocol was approved by the Local Ethics Committee and all patients gave their written informed consent.

\section{Methods}

\section{Sociodemographic data form}

For each patient in the diabetes mellitus and cancer disease groups, data on age, sex, background, marital status, education level, religion were collected.

\section{Temperament and Character Inventory (TCI)}

Each participant in the study was administered the Temperament and Character Inventory (TCI) for evaluation of personality traits. TCI is a self-administered questionnaire that measures the 4 temperament dimensions (NS, HA, RD, and PS) and the 3 character dimensions (SD, CO, and ST). The 240 items are answered "yes" or "no". 
Each temperament and character dimension, except for persistence, is evaluated as the sum of scores of 3 up to 5 subscales that measure more specific traits. The subscales corresponding to each scale were analyzed.

\section{Hospital Anxiety and Depression Scale (HADS)}

HADS is a 14-item scale which determines the generation of ordinal data. Of the 14 items, 7 assess anxiety and the other 7, depression. Each item is scored from 0 to 3. Both anxiety and depression are considered absent at a score lower than 7 , moderately present at a score between 8-10, and major at a score higher than 11.

HADS is a practical screening tool for identifying and quantifying anxiety and depression in the medical clinic for non-psychiatric patients. The Romanian version of the scale shows appropriate reliability and validity. The use of this instrument is strongly recommended to assess anxiety and depression in the chronic pain population, as it does not include items of a somatic nature that can give false positive results. In Romania, the validity of HADS was confirmed in both psychiatric [22] and medical adult patients [23] and the validation studies showed a high internal consistency of both scales: HADS-A (Cronbach's $\alpha$ ranging between .68 and .93) and HADS-D (Cronbach's $\alpha$ ranging between .67 and .90) [23].

\section{Brief Pain Inventory (BPI)}

Brief Pain Inventory (BPI) is a self-administered questionnaire originally designed to assess cancer pain, which is now also used as a generic pain questionnaire for chronic pain. It is available in a short (9 items) and long (17 items) form. The short BPI form is the most frequently used. The first, optional, item is a screening question about the respondent's pain on the day. The questionnaire is then composed of pain drawing diagrams, four items about pain intensity (worst pain, least pain, average pain, pain right now), two items on pain relief treatment or medication, and one item on pain interference, with seven subitems (general activity, mood, walking ability, normal walk, relations with other people, sleep, and enjoyment of life) [24]. A consensus panel recommended that the two domains measured by BPI - pain intensity and the impact of pain on functioning (interference), be included as outcomes in all chronic-pain clinical trials [25].

Data collection was performed using the method of non-probabilistic, rational sampling conditioned by the inclusion and exclusion criteria presented above.

\section{Statistical analysis}

Statistical analysis was carried out using SPSS 25.0 and Microsoft Excel. The data in this study were described using descriptive statistical methods (means, percentages, and standard deviations). Analyses of variance (ANOVAs) were performed to assess whether each dependent variable of TCI was statistically different between the groups. The level of significance was set at $p<0.001$ (Bonferroni correction). The t-test for independent samples with equal variances and Levene's test for comparison of the results obtained on BPI (interference and severity scores) between the group of patients with diabetes mellitus and those with cancer disease were used.

\section{Results}

The analysis of the 130 patients included in the study showed the predominance of the female sex in all three groups ( $72 \%$ diabetes group, $57 \%$ cancer group, $70 \%$ control group), an average age of 60 in the cancer group, of 54 in the diabetes group and of 39 in the control group.

From a psychosocial point of view, the patients came from urban areas, had secondary education, were married and of Orthodox religion.

\section{Inventory)}

Results of TCI (Temperament and Character

Significant results were obtained for the overall scores of the Harm Avoidance, Reward dependence, SelfDirectedness and Cooperativeness scales and isolated significant scores for the Novelty Seeking (NS1) subscale.

\section{Domains of Temperament}

Results regarding Novelty Seeking (NS), Harm Avoidance (HA), Reward Dependence (RD), Persistence (P) scales and their respective subscales are described in Table I.

No significant difference was obtained for the total mean value of the Novelty Seeking scale, however NS1 subscale had significantly lower scores in both studied groups $(\mathrm{p}<0.001)$.

Harm Avoidance was significantly higher $(\mathrm{p}<0.001)$ in both studied groups. Also, the mean value obtained on subscale HA4 was significantly higher for both studied groups compared to the control group.

The value obtained on the Reward Dependence scale and on the RD3 subscale in both studied groups were significantly lower $(\mathrm{p}<0.001)$ than in the control group.

No significant difference was found on the Persistence $(\mathrm{P})$ scale between the 3 groups.

\section{Domains of Character}

Results regarding Self-Directedness (SD), Cooperativeness (CO), and Self-Transcendence (ST) and their respective subscales are represented in Table II.

Self-Directedness (SD) had significantly lower scores $(p<0.001)$ in both studied groups compared to the control group. Furthermore SD1 and SD3 had significantly lower scores in the studied groups, in contrast to SD2 that had significantly lower scores only in the diabetes mellitus group.

Cooperativeness (CO) values were significantly lower for the subjects with cancer and those with diabetes $(p<0.001)$, as were the values for the C2 and C5 subdimensions compared to the control group. Furthermore $\mathrm{C} 1$ and the $\mathrm{C} 3$ subscales had significantly lower scores only in the diabetes mellitus group.

The Self-Transcendence (ST) scale showed no significant difference for the total mean value between the 3 groups. 
Table I. Temperament domains of individuals with diabetes mellitus or cancer disease compared with control group.

\begin{tabular}{|c|c|c|c|c|c|}
\hline & $\begin{array}{c}\text { DM Group } \\
(\text { Mean } \pm \text { SE) }\end{array}$ & $\begin{array}{c}\text { CD Group } \\
(\text { Mean } \pm \text { SE) }\end{array}$ & $\begin{array}{c}\text { CON Group } \\
(\text { Mean } \pm \text { SE) }\end{array}$ & $\begin{array}{c}\text { DM vs CON } \\
(\text { ANOVA) }\end{array}$ & $\begin{array}{c}\text { CD vs CON } \\
(\text { ANOVA) }\end{array}$ \\
\hline Novelty Seeking (NS) Total & $15.26 \pm 3.99$ & $15.35 \pm 5.28$ & $17.57 \pm 5.63$ & .025 & .072 \\
\hline Exploratory excitability vs. stoic rigidity (NS1) & $4.98 \pm 1.86$ & $4.75 \pm 2.26$ & $6.72 \pm 2.26$ & .000 & .000 \\
\hline Impulsiveness vs. reflection (NS2) & $3.48 \pm 1.79$ & $3.67 \pm 2.1$ & $2.97 \pm 2.06$ & .219 & .140 \\
\hline Extravagance vs. reserve (NS3) & $3.58 \pm 1.48$ & $3.60 \pm 1.99$ & $4.57 \pm 1.99$ & .008 & .032 \\
\hline Disorderliness vs. regimentation (NS4) & $3.30 \pm 1.74$ & $3.22 \pm 1.74$ & $3.22 \pm 1.75$ & .830 & .949 \\
\hline Harm avoidance (HA) Total & $19.64 \pm 6.20$ & $19.85 \pm 7.63$ & $13.32 \pm 6.02$ & .000 & .000 \\
\hline Worry/pessimism vs. uninhibited optimism (HA1) & $5.54 \pm 2.55$ & $5.60 \pm 2.68$ & $4.05 \pm 2.14$ & .004 & .006 \\
\hline Fear of uncertainty vs. confidence & $4.72 \pm 1.40$ & $4.85 \pm 2.01$ & $3.87 \pm 1.58$ & .009 & .019 \\
\hline Shyness with strangers vs. gregariousness (HA3) & $4.04 \pm 1.64$ & $4.27 \pm 2.30$ & $3.05 \pm 2.01$ & .012 & .013 \\
\hline Fatigability/asthenia vs. vigor (HA4) & $5.34 \pm 2.20$ & $5.12 \pm 2.26$ & $2.40 \pm 2.09$ & .000 & .000 \\
\hline Reward dependence (RD) Total & $14.34 \pm 3.5$ & $14.35 \pm 3.23$ & $17.35 \pm 3.2$ & .000 & .000 \\
\hline Sentimentality vs. insensitivity (RD1) & $7.00 \pm 1.87$ & $7.27 \pm 2.13$ & $7.80 \pm 1.66$ & .037 & .224 \\
\hline Attachment vs. detachment (RD3) & $4.60 \pm 1.96$ & $4.35 \pm 1.71$ & $6.27 \pm 1.81$ & .000 & .000 \\
\hline Dependence vs. independence (RD4) & $2.74 \pm 1.74$ & $2.72 \pm 1.37$ & $3.27 \pm 1.48$ & .127 & .090 \\
\hline Persistence (P) Total & $4.18 \pm 1.40$ & $4.15 \pm 1.44$ & $4.55 \pm 1.53$ & .238 & .331 \\
\hline
\end{tabular}

Table II. Character domains of individuals with diabetes mellitus or cancer disease compared with control group

\begin{tabular}{|c|c|c|c|c|c|}
\hline & $\begin{array}{c}\text { DM Group } \\
(\text { Mean } \pm \text { SE) }\end{array}$ & $\begin{array}{c}\text { CD Group } \\
\text { (Mean } \pm \text { SE) }\end{array}$ & $\begin{array}{l}\text { CON Group } \\
(\text { Mean } \pm \text { SE })\end{array}$ & $\begin{array}{c}\text { DM vs CON } \\
\text { (ANOVA) }\end{array}$ & $\begin{array}{c}\text { CD vs CON } \\
(\text { ANOVA) }\end{array}$ \\
\hline Self-Directedness (SD) Total & $27.30 \pm 6.38$ & $29.30 \pm 4.91$ & $34.60 \pm 3.7$ & .000 & .000 \\
\hline Responsibility vs. blaming (SD1) & $5.08 \pm 1.95$ & $4.95 \pm 1.41$ & $6.82 \pm 1.05$ & .000 & .000 \\
\hline Purposefulness vs. lack of goal direction (SD2) & 4. $80 \pm 1.60$ & $5.47 \pm 1.58$ & $6.22 \pm 1.42$ & .000 & .029 \\
\hline Resourcefulness vs. inertia (SD3) & $2.60 \pm 1.21$ & $2.82 \pm 1.33$ & $4.50 \pm 0.75$ & .000 & .000 \\
\hline Self-acceptance vs. self-striving (SD4) & $6.96 \pm 2.25$ & $7.47 \pm 2.02$ & $6.72 \pm 2.59$ & .647 & .153 \\
\hline Cooperativeness $(\mathrm{CO})$ Total & $28.7 \pm 5.25$ & $30.1 \pm 4.17$ & $35.02 \pm 3.05$ & .000 & .000 \\
\hline Social acceptance vs. social intolerance (C1) & $5.58 \pm 1.84$ & $6.25 \pm 1.54$ & $7.12 \pm 1.01$ & .000 & .004 \\
\hline Empathy vs. social disinterest (C2) & $3.66 \pm 1.39$ & $3.72 \pm 1.53$ & $5.35 \pm 0.89$ & .000 & .000 \\
\hline Helpfulness vs. unhelpfulness (C3) & $5.48 \pm 1.35$ & $6.02 \pm 1.02$ & $6.75 \pm 1.29$ & .000 & .007 \\
\hline Compassion vs. revengefulness $(\mathrm{C} 4)$ & $7.78 \pm 1.58$ & $7.92 \pm 1.24$ & $8.37 \pm 1.14$ & .049 & .097 \\
\hline Pure-hearted principles vs. self-advantage (C5) & $6.24 \pm 1.49$ & $6.22 \pm 1.18$ & $7.47 \pm 1.08$ & .000 & .000 \\
\hline Self-transcendence (ST) Total & $16.28 \pm 6.62$ & $16.85 \pm 5.68$ & $14.15 \pm 6.34$ & .126 & .048 \\
\hline Self-forgetful vs. self-conscious (ST1) & $5.40 \pm 2.55$ & $5.50 \pm 2.43$ & $3.82 \pm 2.17$ & .003 & .002 \\
\hline Transpersonal identification vs. self-differentiation (ST2) & $4.70 \pm 2.34$ & $5.22 \pm 2.15$ & $3.12 \pm 2.05$ & .001 & .000 \\
\hline Spiritual acceptance vs. rational Materialism (ST3) & $6.18 \pm 2.94$ & $6.12 \pm 2.77$ & $7.22 \pm 3.25$ & .114 & .108 \\
\hline
\end{tabular}


Results following the application of HADS (Hospital Anxiety and Depression Scale)

Following quantification of the anxiety and depression forms in the two study groups using the HADS scale, it was observed that major anxiety was more frequent in diabetics (34\%) compared to a much lower percentage $(20 \%)$ in cancer patients. Moderate anxiety was found in almost equal proportions in the two groups $(22 \%)$. The absence of anxiety was more frequent in the group of cancer patients $(57.5 \%)$ compared to the group of diabetic patients (44\%). Regarding depression, the major form was seen in $17.5 \%$ of cancer patients compared to $16 \%$ of diabetics. Moderate depression was more frequent in diabetics (24\%) compared to cancer patients (15\%), and the absence of depression was more frequent in cancer patients $(67.5 \%)$ compared to diabetics $(60 \%)$

\section{Pain Inventory)}

Following analysis of the BPI inventory, higher severity and interference scores were obtained for the group of diabetic patients (Table III). The differences between the two groups were significant regarding the BPI severity score (t-test for independent samples, $\mathrm{p}=0.03$ ) (Levene's test for equal variances $\mathrm{F}=0.539, \mathrm{p}=0.465$, t-test for independent samples with equal variances, $\mathrm{t}=2.205, \mathrm{p}=0.03$ ) (Table IV). In the case of the interference score, there were no significant differences between the two groups (Levene's test $\mathrm{F}=2.63, \mathrm{p}>0.05$, t-test for independent samples with equal variances, $\mathrm{t}=1.11, \mathrm{p}>0.05$ ).

Table III. Mean and standard deviation $(\mathrm{m} \pm \mathrm{sd})$ for BPI scores.

\begin{tabular}{l|l|l|l|l} 
Score & Cancer & DM & p (Levene) & p (t-test) \\
\hline BPI Severity Score & $3.42 \pm 2.55$ & $4.54 \pm 2.25$ & 0.39 & 0.03 (equal variances) \\
BPI Interference Score & $4.11 \pm 2.83$ & $4.71 \pm 2.33$ & 0.19 & 0.267 (equal variances)
\end{tabular}

Table IV. Mean and standard deviation, t-test and Levene's test for the BPI severity score.

\section{Group Statistics}

\begin{tabular}{|l|c|c|c|c|}
\hline & N & Mean & Std. Deviation & Std. Error Mean \\
\hline Diabetes Mellitus Patients & 50 & 4.5440 & 2.25082 & 0.31831 \\
Oncology Patients & 40 & 3.4250 & 2.55842 & 0.40452 \\
\hline
\end{tabular}

Independent Samples Test

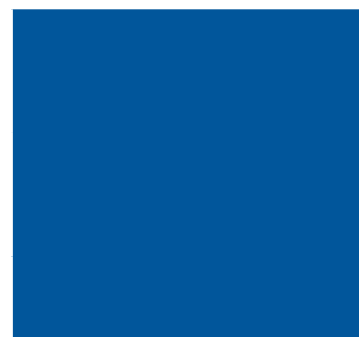

BPI Severity Score Equal variances assumed

Equal variances not assumed

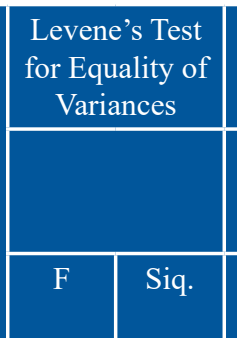

.539

.465

2.205

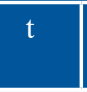

Df

Siq. (2-tailed)

88

.030

$2.174 \quad 78.343$

.033

1.11900

.51474

.09429

2.14371
.033

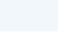




\section{Discussion}

In this investigation, we have found that individuals with diabetes and cancer have significantly higher scores in the scale of Harm Avoidance and significantly lower scores in the Self-Directedness, Reward Dependence and Cooperativeness scales when compared to the to age- and gender-matched controls.

The findings of the current study suggest that personality traits can have a determinant role in integrating the concept of disease and this could explain the variability of pain perception among individuals. Even more, this study indicates that patients who experience pain may exhibit a mutual personality profile featuring high levels of Harm Avoidance accompanied by low levels of SelfDirectedness, as mentioned in other studies [26,27]. In addition, our findings point out that more attention should be paid to the low scores in Reward Dependence and Cooperativeness of cancer and diabetes patients.

Harm Avoidance assessment could be useful in clinical practice as a high score predicts a negative perception of pain, a distorted representation of the disease and a worse response to antidepressant treatment in mood disorders $[28,29]$.

Higher scores of Harm Avoidance in patients with diabetes and cancer compared to the control group suggest that these individuals could be described as cautious, tense, fearful, worrying, passive and fatigable. These traits are considered to be markers of vulnerability [30,31] of a higher severity of depression [32] but also of pain-related anxiety [33]. Furthermore, Harm Avoidance is regarded as the TCI equivalent of behavioral inhibition [32], which is considered to reflect sensitivity to stress on a behavioral level [34]. On the HA scale, significantly higher values were obtained for subscale HA4 (Fatigability and Asthenia vs Vigor). According to Cloninger's model, with high Fatigability recover from minor illnesses and stress more slowly than average people individuals do [18]. Fatigability as a trait construct reflects the negative affectivity associated with an enhanced distress in somatic illnesses.

Low scores, as the ones we obtained on the SelfDirectedness scale, reflect an individual's tendency to blame other people and external circumstances for what is happening to them (SD1), alongside their struggle to find direction, purpose and meaning in their lives (SD2). As a consequence they generally seem to be lacking an internal locus of control, which prevents them to define, set and pursue meaningful goals [18].

Our results indicate lower scores in Reward Dependence especially in subscale RD3 in patients with diabetes and cancer. This suggests that they are reserved individuals who don't tend to express their emotions and energy, behavior that has a negative impact on the perception of pain and disease. The low scores in Cooperativeness scale indicate the fact that these patients tend to retreat and to not ask for help, even if they are in distress. Further research should be conducted for a better understanding of the low Reward Dependence and Cooperativeness scores in patients with diabetes mellitus or cancer disease.

The low scores obtained on the Novelty Seeking scale suggest that patients in the diabetes mellitus and cancer disease groups have a tendency to be slow, indifferent, to lack curiosity and enthusiasm.

Pain and emotions are frequently associated with multiple comorbid symptoms [35]. The perception of pain is accompanied by anxiety, depression and other emotional symptoms $[3,4]$. Our research shows that major anxiety was more frequent in patients with diabetes. Diabetes is a growing problem that poses a major public health challenge globally. Patients with diabetes are more predisposed to depression [36,37], anxiety and personality disorders, and at the same time these conditions can increase the risk of developing diabetes. Depression is the most commonly researched factor in studies regarding diabetes. However, more research should be conducted to investigate anxiety disorders in these patients. Overviews of a study [38] suggest that diabetes is associated with $20 \%$ increased odds of having an anxiety disorder and $48 \%$ increased odds of having elevated anxiety symptoms. This elevated anxiety could be the reason why pain perception is frequently more intense in diabetes patients, as reflected in the high scores obtained in the BPI.

Major depression was more frequent in patients with cancer. It is known that both pain and depression are common in cancer patients and many studies have indicated a significant association between these two prevalent symptoms in these patients $[39,40]$.

Even though the prevalence of chronic pain in cancer free patients is high, it remains underappreciated in comparison with the quest for survival [41]. Patients with a long history of pain disorders have increased anxiety and depression symptoms, as well as suicidal thoughts. A regular basic evaluation can help oncology staff to identify patients with clinical depression. There has been an extensive discussion in literature about the fact that patients suffering from chronic diseases and pain associated with anxiety and depression have poor emotional regulation strategies [1]. It would be more important to know the underlying mechanism of the two comorbidities- pain and emotional problems (anxiety and depression) than to focus on diagnostic and treatment modalities alone, in the hope that treating one condition can improve the other.

The limitations of this study are the relatively small number of patients, reliance on self-reported data, and the patients' heterogeneous pain disorders that can complicate the interpretation of the results. HA scores may be susceptible to demographic factors such as gender, age and educational level.

The strengths of the study reside in there not being many studies that investigate the link between personality dimensions and anxiety in patients with diabetes or cancer 
who experience pain. Furthermore, our results were significant in the Reward Dependence and Cooperativeness scales, not just in the Harm Avoidance and Self-Directedness scales as previously studied.

\section{Conclusions}

This study reveals that there is a link between personality dimensions and pain perception in patients with diabetes mellitus or cancer and emphasizes the traits that make them vulnerable when confronting pain as well as their disease. Our results confirm the existence of a mutual personality profile featuring high levels of Harm Avoidance and low levels of Self-Directedness in patients who experience pain, as previously studied. More attention should be paid to associated anxiety in patients with diabetes mellitus or neoplasms who experience pain, whose personality is insufficiently investigated and is a major factor of treatment resistance. Our finding regarding low levels of Reward Dependence and Cooperativeness could potentially extend the personality profile of these patients, and open a gateway to future research.

\section{References}

1. Linton SJ. A Transdiagnostic Approach to Pain and Emotion. J Appl Biobehav Res. 2013;18:82-103.

2. Trost Z, Vangronsveld K, Linton SJ, Quartana PJ, Sullivan MJ. Cognitive dimensions of anger in chronic pain. Pain. 2012; 153:515-517.

3. Asmundson GJ, Abramowitz JS, Richter AA, Whedon M. Health anxiety: current perspectives and future directions. Curr Psychiatry Rep. 2010;12:306-312.

4. Asmundson GJ, Katz J. Understanding the co-occurrence of anxiety disorders and chronic pain: state-of-the-art. Depress Anxiety. 2009;26:888-901.

5. Leeuw M, Goossens ME, Linton SJ, Crombez G, Boersma K, Vlaeyen JW. The fear-avoidance model of musculoskeletal pain: current state of scientific evidence. J Behav Med. 2007;30:77-94.

6. Vlaeyen JW, Linton SJ. Fear-avoidance model of chronic musculoskeletal pain: 12 years on. Pain. 2012;153:1144-1147.

7. Eccleston C, Crombez G. Worry and chronic pain: a misdirected problem solving model. Pain. 2007;132:233-236.

8. Sullivan MJ, Rodgers WM, Kirsch I. Catastrophizing, depression and expectancies for pain and emotional distress. Pain. 2001;91:147-154.

9. Pulvers K, Hood A. The role of positive traits and pain catastrophizing in pain perception. Curr Pain Headache Rep. 2013; 17:330.

10. Cloninger CR, Svrakic DM, Przybeck TR. A psychobiological model of temperament and character. Arch Gen Psychiatry. 1993;50:975-990.

11. Jylhä $P$, Isometsä E. Temperament, character and symptoms of anxiety and depression in the general population. Eur Psychiatry. 2006;21:389-395.

12. Moresco FM, Dieci M, Vita A, Messa C, Gobbo C, Galli
L, et al. In vivo serotonin $5 \mathrm{HT}(2 \mathrm{~A})$ receptor binding and personality traits in healthy subjects: a positron emission tomography study. Neuroimage. 2002;17:1470-1478.

13. Conrad R, Schilling G, Bausch C, Nadstawek J, Wartenberg $\mathrm{HC}$, Wegener I, et al. Temperament and character personality profiles and personality disorders in chronic pain patients. Pain. 2007;133:197-209.

14. Cloninger CR. A unified biosocial theory of personality and its role in the development of anxiety states. Psychiatr Dev. 1986;4:167-226.

15. Waddell G, Newton M, Henderson I, Somerville D, Main CJ. A Fear-Avoidance Beliefs Questionnaire (FABQ) and the role of fear-avoidance beliefs in chronic low back pain and disability. Pain. 1993;52:157-168.

16. Asmundson GJ, Norton PJ, Norton GR. Beyond pain: the role of fear and avoidance in chronicity. Clin Psychol Rev. 1999;19:97-119.

17. Vlaeyen JW, Linton SJ. Fear-avoidance and its consequences in chronic musculoskeletal pain: a state of the art. Pain. 2000;85:317-332.

18. Cloninger $\mathrm{CR}$. The temperament and character inventory (TCI): a guide to its development and use. St. Louis, Mo.: Center for Psychobiology of Personality, Washington University; 1994.

19. Boz C, Velioglu S, Ozmenoglu M, Sayar K, Alioglu Z, Yalman B, et al. Temperament and character profiles of patients with tension-type headache and migraine. Psychiatry Clin Neurosci. 2004;58:536-543.

20. Mongini F, Fassino S, Rota E, Deregibus A, Levi M, Monticone D, et al. The temperament and character inventory in women with migraine. J Headache Pain. 2005;6:247-249.

21. Malmgren-Olsson EB, Bergdahl J. Temperament and character personality dimensions in patients with nonspecific musculoskeletal disorders. Clin J Pain. 2006;22:625-631.

22. Ladea M. validarea scalei de anxietate si depresie (hads) pe o populatie de pacienti psihiatrici din tara noastra [Validation of the hospital anxiety and depression scale (hads) in a population of psychiatric patients in Romania]. Available from: http://www.romjpsychiat.ro/article/validarea-scaleide-anxietate-si-depresie-hads-pe-o-populatie-de-pacientipsihiatrici-din-tara-noastr-maria-ladea

23. De Smedt D, Clays E, Doyle F, Kotseva K, Prugger C, Pająk A, et al. Validity and reliability of three commonly used quality of life measures in a large European population of coronary heart disease patients. Int J Cardiol. 2013;167:2294-2299.

24. Poquet N, Lin C. The Brief Pain Inventory (BPI). J Physiother. 2016;62:52.

25. Turk DC, Dworkin RH, Allen RR, Bellamy N, Brandenburg $\mathrm{N}$, Carr DB, et al. Core outcome domains for chronic pain clinical trials: IMMPACT recommendations. Pain. 2003;106:337-345.

26. Naylor B, Boag S, Gustin SM. New evidence for a pain personality? A critical review of the last 120 years of pain and personality. Scand J Pain. 2017;17:58-67.

27. Gustin SM, Burke LA, Peck CC, Murray GM, Henderson LA. Pain and Personality: Do Individuals with Different 
Forms of Chronic Pain Exhibit a Mutual Personality? Pain Pract. 2016;16:486-494.

28. Abrams KY, Yune SK, Kim SJ, Jeon HJ, Han SJ, Hwang $\mathrm{J}$, et al. Trait and state aspects of harm avoidance and its implication for treatment in major depressive disorder, dysthymic disorder, and depressive personality disorder. Psychiatry Clin Neurosci. 2004;58:240-248.

29. Mulder RT, Joyce PR, Frampton CM, Luty SE, Sullivan PF. Six months of treatment for depression: outcome and predictors of the course of illness. Am J Psychiatry. 2006; 163:95-100.

30. M.D CRC. Feeling Good: The Science of Well-Being. Oxford, New York: Oxford University Press; 2004: pp. 400.

31. Cloninger CR, Svrakic DM, Przybeck TR. Can personality assessment predict future depression? A twelve-month follow-up of 631 subjects. J Affect Disord. 2006;92:35-44.

32. Kampman O, Poutanen O. Can onset and recovery in depression be predicted by temperament? A systematic review and meta-analysis. J Affect Disord. 2011;135:20-27.

33. Knaster P, Estlander AM, Karlsson H, Kaprio J, Kalso E. Temperament traits and chronic pain: the association of harm avoidance and pain-related anxiety. PLoS One. 2012;7:e45672.

34. Tyrka AR, Wier LM, Price LH, Rikhye K, Ross NS, Anderson GM, et al. Cortisol and ACTH responses to the
Dex/CRH test: influence of temperament. Horm Behav. 2008;53:518-525.

35. Harvey AG. Insomnia, psychiatric disorders, and the transdiagnostic perspective. Curr Dir Psychol Sci. 2008;17:299-303.

36. Breivik H, Collett B, Ventafridda V, Cohen R, Gallacher D. Survey of chronic pain in Europe: prevalence, impact on daily life, and treatment. Eur J Pain. 2006;10:287-333.

37. de Jonge P, Rosmalen JG. Comment on: Knol MJ, Twisk JWR, Beekman ATF, Heine RJ, Snoek FJ, Pouwer F. (2006) depression as a risk factor for the onset of type 2 diabetes mellitus. A meta-analysis. Diabetologia; 49: 837-845. Diabetologia. 2006;49:2797-2798; author reply 2799-2800.

38. Mezuk B, Eaton WW, Albrecht S, Golden SH. Depression and type 2 diabetes over the lifespan: a meta-analysis. Diabetes Care. 2008;31:2383-2390.

39. Akechi T, Okamura H, Nishiwaki Y, Uchitomi Y. Psychiatric disorders and associated and predictive factors in patients with unresectable nonsmall cell lung carcinoma: a longitudinal study. Cancer. 2001;92:2609-2622.

40. Spiegel D. Cancer and depression. Br J Psychiatry Suppl. 1996;(30):109-116.

41. Burton AW, Fanciullo GJ, Beasley RD, Fisch MJ. Chronic pain in the cancer survivor: a new frontier. Pain Med. 2007;8:189-198. 